\title{
Anthony J. Steinbock, Moral Emotions. Reclaiming the Evidence of the Heart
}

Evanston, Illinois, Northwestern University, coll. « Studies in Phenomenology and Existential Philosophy », 2014, 339 pages

\section{Claudia Serban}

\section{(2) OpenEdition}

Journals

Édition électronique

URL : http://journals.openedition.org/alter/441

DOI : $10.4000 /$ alter.441

ISSN : 2558-7927

Éditeur :

Association ALTER, Archives Husserl (CNRS-UMR 8547)

Édition imprimée

Date de publication : 1 décembre 2016

Pagination : 259-265

ISBN : 978-2-9550449-2-6

ISSN : 1249-8947

Référence électronique

Claudia Serban, «Anthony J. Steinbock, Moral Emotions. Reclaiming the Evidence of the Heart », Alter [En ligne], 24 | 2016, mis en ligne le 01 décembre 2016, consulté le 23 septembre 2020. URL : http:// journals.openedition.org/alter/441; DOI : https://doi.org/10.4000/alter.441

Ce document a été généré automatiquement le 23 septembre 2020.

Revue Alter 


\section{Anthony J. Steinbock, Moral Emotions. Reclaiming the Evidence of the Heart}

Evanston, Illinois, Northwestern University, coll. « Studies in Phenomenology and Existential Philosophy », 2014, 339 pages

Claudia Serban

\section{RÉFÉRENCE}

Anthony J. Steinbock, Moral Emotions. Reclaiming the Evidence of the Heart, Evanston, Illinois, Northwestern University, coll. « Studies in Phenomenology and Existential Philosophy », 2014, 339 pages 
1 Le dernier livre d'Anthony Steinbock, Moral Emotions, aborde une thématique en apparence inédite chez cet auteur connu aux spécialistes de phénoménologie surtout pour Home and Beyond: Generative Phenomenology after Husserl (1995), mais aussi, plus récemment, pour Phenomenology and Mysticism. The Verticality of Religious Experience (2007) ; et cependant, il investit et prolonge les résultats de ces ouvrages à plus d'un titre, pour révéler ce faisant la profonde cohérence du travail de son auteur. La question abordée présente néanmoins des défis singuliers qui prescrivent une façon de procéder relativement nouvelle : Moral Emotions met ainsi en œuvre une méthodologie bien particulière qui allie la description phénoménologique (en

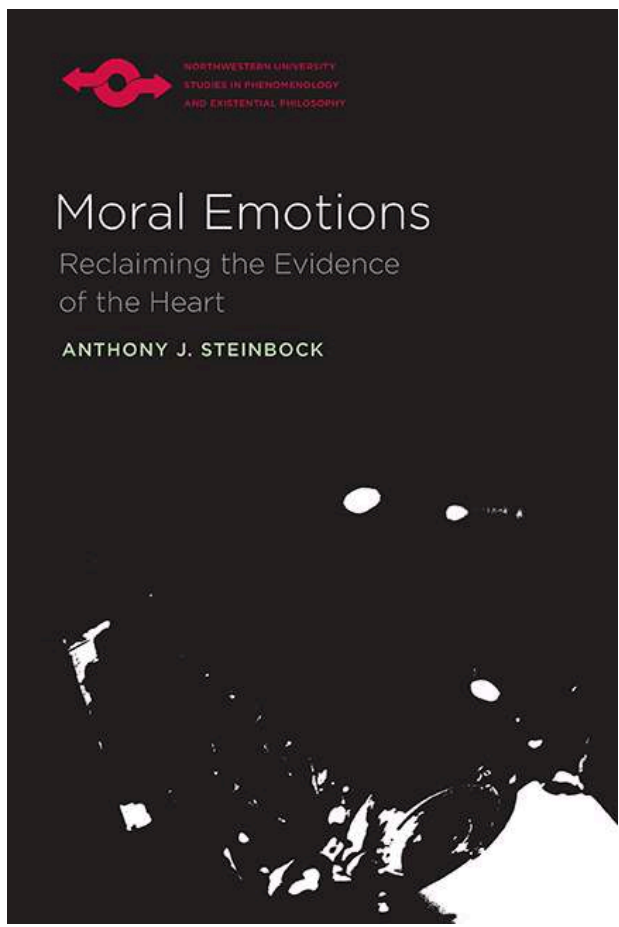
première, deuxième et troisième personne), l'analyse eidétique, l'interprétation de pratiques langagières quotidiennes et le recours à des exemples réels comme fictifs, afin de proposer ce que l'on pourrait appeler une odyssée de la vie morale, allant de l'orgueil à l'humilité. La construction du livre est en effet remarquable en elle-même, et nous aurons l'occasion d'y revenir; relevons pour l'instant le fait qu'elle est mue par un rigoureux effort de catégorisation qui conduit à distinguer, mais aussi à articuler, les émotions morales de la donation à soi (ou de l'auto-donation, self-givenness), en première partie, les émotions morales de la possibilité (deuxième partie) et celles de l'altérité (troisième partie).

2 L'Introduction de l'ouvrage défend d'entrée de jeu la thèse du caractère distinct (distinctiveness) des émotions morales - comprises comme «émotions qui sont essentiellement interpersonnelles, ou qui surgissent dans le rapport interpersonnel » (p.12) -, pour affirmer non seulement qu'elles ne sauraient être dénoncées comme opaques ou confuses, mais aussi qu'elles ont une forme d'évidence sui generis, que l'auteur appelle, d'une formule schelérienne à résonance pascalienne, l'évidence du cœur (the evidence of the heart). En vertu de leur évidence propre, les émotions morales détiennent en outre une fonction de révélation de la personne humaine (p. 11) - autrement dit, elles dévoilent ce qu'est la personne, fût-elle comprise en général comme «le mouvement et l'orientation dynamique qui vit à travers des actes » (p.11) ou fût-elle prise dans sa singularité. La description des émotions morales se situe ainsi au niveau d'une généralité d'expérience qui ne prétend à aucun moment s'identifier à un universel de nature éthique. (La Conclusion reviendra d'ailleurs sur ce point important : décrire les structures essentielles de l'expérience morale ne revient pas à lui imposer des normes ou à nier son enracinement historique et culturel.)

3 Les émotions morales de la donation à soi analysées par la première partie de l'ouvrage sont au nombre de trois : l'orgueil (pride, terme plus polysémique en anglais dans la mesure où il désigne également la fierté), la honte (là encore, le terme anglais shame est 
plus vaste, pour autant qu'il embrasse aussi les manifestations de la pudeur) et la culpabilité (guilt). Parmi elles, l'orgueil (chapitre 1) se caractérise de façon singulière par sa « résistance à l'altérité », par le fait qu'il ne reconnaît autrui qu'en le tenant à l'écart, en niant son rôle dans la constitution de la personne : dans l'orgueil, je me pose comme "l'unique ou la plus haute source du sens» (p. 35). C'est là le paradoxe constitutif de l'orgueil: il ne peut émerger qu'en contexte interpersonnel, et pourtant toute sa signification revient à nier ce contexte qui le fait émerger ( $c f$. p. 36). Comment, dès lors, dépasser l'orgueil et s'ouvrir à l'altérité ? En essayant d'élaborer une réponse à cette question, l'un des apports les plus originaux de l'ouvrage d'Anthony Steinbock consiste dans la reconduction des émotions morales à leurs " racines esthétiques » (p.40), à savoir à certaines manières fondamentales de s'inscrire dans l'espace et dans le temps. Dans cette perspective, la forme la plus élémentaire d'orgueil est représentée par l'orgueil « esthétique » provenant du fait de se poser comme la source privilégiée de la temporalisation et de la spatialisation, ce qui va de pair avec le refus implicite d'une temporalité générative et d'un monde commun. Dès lors, la dissolution de l'orgueil véritable "réduction morale" (p.51) - ne sera pas un acte que j'initie, mais elle adviendra au moment où le paradoxe inhérent à l'orgueil s'avérera comme intenable : autrui est là même si je lui résiste, et la temporalisation et la spatialisation trouvent leur source en dehors de moi, dans l'horizon d'une histoire et d'un monde communs, même si j'essaie de les résorber dans la sphère du propre.

Dans l'orgueil, la révélation à soi de la personne ne saurait donc être complète dans la mesure où elle occulte l'horizon constitutif de l'altérité : une auto-donation véritable ne devient possible que lorsque le moment de l'orgueil est dépassé, comme cela arrive par exemple dans la honte (chapitre 2). La honte peut en effet déclencher une « réduction morale » et contribuer à mettre en question le moi orgueilleux. Cette mise en question n'est cependant pas à comprendre comme une destitution sans reste car, comme le souligne l'auteur, «la honte n'est pas possible sans un véritable amour de soi et une évaluation de soi positive"(p.77). La description phénoménologique des manifestations de la honte est entrelacée ici avec une discussion serrée avec Hegel, Scheler, Sartre et Agamben, qui conduit à identifier dans la honte un des commencements possibles de la vie morale (p. 85), ainsi qu'un des paradigmes les plus féconds de la critique de soi (en lien avec la question de la vocation, chère à l'auteur). Le chapitre se conclut ainsi avec l'affirmation d'un «modèle distinct de normativité qui émerge dans le rapport interpersonnel » (p. 99) s'attestant dans la honte.

5 Tout comme la honte, la culpabilité (chapitre 3) a le pouvoir de dissoudre l'orgueil et de déclencher la critique morale de soi : elle me fait en effet apparaitre à moi-même en me convoquant devant autrui et en me dévoilant comme responsable. Ainsi comprise, la culpabilité est tout sauf une dette ou une faute impersonnelle et indéterminée : sur ce point, l'auteur se démarque résolument de l'analyse du Schuldigsein proposée par Heidegger dans Être et temps, en insistant sur la nécessité d'individualiser ou de singulariser la faute au moyen de la prise en compte de la "vocation personnelle " (p.124). Cette référence à une expérience fondamentale de la vocation, unique pour chaque personne, est un des endroits où transparaît le fait que les émotions morales ont intrinsèquement une dimension religieuse (cf. p. 128), dimension qui signifie que le rapport interpersonnel réalisé dans l'expérience morale est aussi, implicitement ou potentiellement, rapport à une Personne transcendante absolue. (Dans la Conclusion, l'auteur prendra par ailleurs soin d'insister sur le fait que cette signification religieuse est irréductible à tout fondamentalisme ou fanatisme religieux, et inscrira ouvertement 
son entreprise dans le sillage de la conception d'une « religion dynamique » et d'une «moralité ouverte» proposée par Bergson dans Les deux sources de la morale et de la religion.)

6 Avec la honte et la culpabilité s'ouvre l'horizon d'une régénération, d'une renaissance morale : de son orientation vers un passé dont il tente de s'absoudre, le moi se tourne ainsi progressivement vers l'avenir de la réparation. C'est ainsi que commencent à prendre contour les émotions morales de la possibilité : le repentir (repentance) et l'espérance (hope). Nous voyons ici combien importante est pour l'auteur la teneur ou l'orientation temporelle des émotions morales : les différentes étapes de l'odyssée de la vie morale nous confrontent aussi à des modèles différents de temporalisation. Ainsi, si l'orgueil se tient au présent et la honte et la culpabilité regardent vers le passé, le repentir et l'espérance invitent à s'ouvrir à l'avenir. L'analyse du repentir proposée au chapitre 4 est à cet égard bien originale, dans la mesure où elle situe le centre de gravité de cette émotion morale non pas dans la rumination du passé mais dans l'orientation «vers l'avenir» (p. 140): le repentir n'est véritable que s'il libère, en modifiant « le sens du passé » et en ouvrant par là « la possibilité d'un avenir qui n'est pas lié par le passé » (p. 146). Le sens profond du repentir est donc d'être une «liberté pour la transformation de soi (freedom for self-revision) » (p. $147 ; c f$. p. 158). Resitué dans l'horizon de la régénération morale, le repentir dévoile à son tour sa signification religieuse intrinsèque, du fait de sa tendance à confronter celle ou celui qui le ressent à l'infini et à l'absolu (cf. p. 154). Ainsi, la dimension morale et la dimension religieuse du repentir apparaissent comme indivises: le repentir est un mouvement d'infinitisation qui repousse toute limite et vise la réconciliation ultime avec soi et avec les autres ( $c f$. p. 158).

7 De cette caractérisation se dégage déjà une parenté insoupçonnée avec l'espérance (chapitre 5), qui partage cette même dynamique d'infinitisation dans laquelle se vit et s'éprouve le possible. Mais le possible ici en question n'est pas le possible de l'imagination ou des constructions de la pensée: c'est un possible d'existence et d'expérience, revers de l'ouverture foncière de la vie de la personne et de sa liberté. L'horizon de possibilité de l'espérance n'est cependant pas illimité et ne saurait aller jusqu'à inclure des possibles contre-factuels, comme cela pourrait encore être le cas pour le désir ou le souhait: la thèse de l'auteur à ce sujet consiste à soutenir que l'espérance ne saurait surgir là où il n'y a aucun «fondement» ou aucune "raison » pour espérer (ground of hope, p. 170). Plus précisément: «je ne peux en même temps espérer et tenir ce qui est espéré pour impossible» (p. 173) : il faut que l'impossible m'apparaisse malgré tout comme "soutenable (sustainable) » afin que je puisse en faire l'objet de mon espoir. Et cependant, ce qui est espéré n'est pas en mon pouvoir, sinon il serait simplement accompli au lieu d'être espéré : c'est pourquoi, en espérant, je m'ouvre nécessairement à une force extérieure, je reconnais une puissance en dehors de moi, une altérité qui peut ici encore se décliner en un Absolu transcendant. L'espérance a donc, tout autant que le repentir ou la culpabilité, une teneur religieuse indéniable (cf. p. 169). Plus loin encore, l'espérance modifie la façon dont je vis le temps : à la différence de l'attente ou de l'anticipation qui se caractérisent par une forme d'impatience, le propre de l'espérance est d'endurer le temps, de le subir avec patience (cf. p. 175). Mais qu'en est-il dès lors du désespoir? Par de très fines analyses, l'auteur montre que ce dernier est à distinguer du manque d'espoir, ou de l'espoir déçu: là où il n'y a plus d'espoir, l'impossible apparaît comme impossible, sans reste ; alors que dans le désespoir, c'est le fondement même de l'espérance qui se dérobe - or 
il ne peut se dérober que pour avoir été aperçu. Par conséquent, « lorsque je désespère, je fais l'expérience d'un avenir fermé aux possibilités pleines de sens qu'il devrait autrement abriter » (p.192) : le possible est alors éprouvé négativement comme ce qui se dérobe à moi.

L'analyse des émotions morales de la possibilité se poursuit avec celle des émotions morales de l'altérité que sont la confiance (trust), l'amour et l'humilité, et qui prolongent singulièrement le mouvement de mise en question de l'orgueil. La confiance (chapitre 6), tout d'abord, est envisagée comme une émotion interpersonnelle dans la mesure où non seulement elle prend tout son sens dans l'horizon du rapport entre personnes, mais elle requalifie en même temps ce rapport : elle en fait, précisément, un « lien de confiance (bond of trust)» (p. 201). La confiance me lie à autrui - littéralement, elle me confie à lui ou à elle, en me décentrant par rapport à moi-même (cf. p. 202). Ce lien est envisagé par l'auteur non pas comme quelque chose d'acquis, de construit, de gagné (comme lorsqu'on dit : «j'ai gagné sa confiance »), ou de fondé rationnellement, mais comme un don libre et gratuit: faire confiance, c'est s'exposer, c'est donc se reconnaître comme vulnérable (p. 207). Le sens de cette vulnérabilité fondamentale peut aller de la dépendance à l'égard d'autrui ou de la faiblesse devant lui (ou devant elle) jusqu'à la finitude qui se révèle dans la confrontation à l'infini, dans la confiance dans cette personne tout autre qu'est Dieu : on voit donc qu'ici encore l'expérience morale a une signification religieuse incontournable. L'auteur insiste en outre sur le fait que la confiance est le liant même de toute communauté sociale pour autant qu'elle a la propriété remarquable de se propager : « la confiance génère la confiance » (p. 213), tel est le principe qui consolide sans cesse les rapports interpersonnels à l'échelle de la société humaine. A ce niveau s'atteste en outre, de façon cruciale, non seulement l'autonomie de la sphère morale par rapport à la sphère théorique qu'affirmait déjà implicitement l'idée d'une évidence sui generis des émotions morales, mais aussi la possibilité d'une fondation des caractères épistémiques dans les caractères moraux: ainsi, la croyance théorique elle-même peut apparaître comme fondée dans la confiance (cf. p. 215). Par ailleurs, loin de s'expliquer par une soumission aux normes sociales, la confiance fonde cette soumission même : ressurgit ainsi l'idée selon laquelle dans les émotions morales s'atteste et s'exprime une normativité propre aux rapports interpersonnels qui ne saurait se laisser dériver de normes sociales déjà constituées.

Le dernier chapitre de Moral Emotions (chapitre 7) conjugue l'analyse de l'amour et celle de l'humilité, tout en annonçant qu'un " traitement phénoménologique plus complet " de la première de ces deux notions est réservé à « un autre ouvrage, qui examinera sa relation à la haine et élaborera l'articulation entre amour érotique, amour sexuel et amour agapique» (p.196). L'amour porte en effet les principaux traits des émotions morales à leur apogée, dans la mesure où il accomplit la « donation de l'aimé » (p. 225) et l'« ouverture aux possibilités les plus profondes» (p. 226) de ce que quelqu'un peut être (là où, par contraste, la haine "ferme les possibilités »). L'amour réalise aussi pleinement l'essence du lien interpersonnel dans la mesure où il consiste à s'ouvrir à l'autre «dans son unicité » (p. 227) tout en supposant « une ouverture à l'infinité » et donc, implicitement, "au divin» (p. 228). Le propre de l'amour est en effet l'illimitation, l'affranchissement à l'égard de toute limite, qui prend d'abord, comme l'auteur le montre, un sens temporel (cf. p. 230) : l'amour se réitère sans cesse au présent, dans un présent qui se veut éternel et qui embrasse donc un passé indéfini et un avenir également sans fin (que la mort même ne saurait donc clôturer). L'humilité, pour sa part, est intrinsèquement liée à l'amour, pour autant qu'elle désigne la manière 
dont je suis donné à moi-même lorsque j'aime véritablement (cf. p. 232). Cela ne veut pourtant pas dire qu'elle advient, comme la honte ou la culpabilité, en réponse à autre chose qu'elle-même : l'humilité ne présuppose pas l'expérience de l'orgueil, elle peut être première et donc fondatrice; mais elle peut aussi, cela va de soi, fonctionner comme une mise en question de l'orgueil, donc comme une forme de «réduction morale ». En cela elle se distingue à la fois de la modestie et de l'humiliation, dont elle ne partage ni le caractère toujours relatif ni la valence négative et qui, en retour, ne participent pas de sa signification temporelle qui est celle d'une "acceptation" (p. 257) sans fin assignée. Dans l'humilité, en effet, je prends ma place dans le temps génératif et dans le monde commun, je suis à la fois présent et reconnaissant (cf. p. 257) et je me dévoue à la «réparation» du monde dans lequel je vis: avec ces constats, l'explicitation de cette dernière émotion morale qui est aussi, en un sens, première, se clôt sur un véritable plaidoyer pour une « écologie » fondée dans l'humilité (cf. p. 260).

$\mathrm{Au}$ terme de ces riches analyses, la Conclusion de Moral Emotions avancera une thèse forte, en affirmant que "s'il y a une "crise", elle ne réside pas dans la Raison, mais dans le fait que les émotions ont été exclues de la signification de la personne » (p. 274), et en prônant la nécessité de "réintégrer explicitement" les émotions morales "dans nos pratiques et discours sociaux et politiques » (p. 275) afin de pouvoir effectuer " une critique du pouvoir et des relations sociales qui sont destructives» (p. 276). Par ces vœux, l'ouvrage d'Anthony Steinbock révèle son indéniable actualité, ainsi que l'ampleur de ses enjeux.

\section{AUTEURS \\ CLAUDIA SERBAN}

Maître de Conférences à l'Université Toulouse 2 Jean Jaurès 